\title{
Universality in W+Multijet Production
}

\section{Zvi Bern, Kemal J. Ozeren}

Department of Physics and Astronomy, UCLA, Los Angeles, CA 90095-1547, USA

E-mail: bernephysics.ucla.edu

\section{Lance J. Dixon, Stefan Höche}

SLAC National Accelerator Laboratory, Stanford University, Stanford, CA 94309, USA

E-mail: LancedsLac.stantord.edu, shoechedsLac.stantord.edu

\section{Fernando Febres Cordero}

Departamento de Física, Universidad Simón Bolívar, Caracas 1080A, Venezuela

E-mail: tEebresdusb.ve

\section{Harald Ita}

Albert-Ludwigs-Universität Freiburg, Physikalisches Institut, D-79104 Freiburg, Germany

E-mail: harald.itadphysik.uni-freiburg.de

\section{David A. Kosower*}

Institut de Physique Théorique, CEA-Saclay, F-91191 Gif-sur-Yvette cedex, France

E-mail: david.kosowerdcea.fr

\section{Daniel Maître}

Institute for Particle Physics Phenomenology, Department of Physics, University of Durham, DH1 3LE, UK

E-mail: daniel maitreddurham.ac.ult

\begin{abstract}
We study $W$-boson production accompanied by multiple jets at $7 \mathrm{TeV}$ at the LHC. We study the jet-production ratio, of total cross sections for $W+n$ - to $W+(n-1)$-jet production, along with the ratio of distributions in the total transverse hadronic jet energy $H_{\mathrm{T}}^{\mathrm{jets}}$. We use these ratios to extrapolate the total cross section, and the differential distribution in $H_{\mathrm{T}}^{\text {jets }}$, to $W+6$-jet production. We use the BLACKHAT software library in conjunction with SHERPA to perform the computations.
\end{abstract}

Loops and Legs in Quantum Field Theory - LL 2014,

27 April - 2 May 2014

Weimar, Germany

\footnotetext{
*Speaker.
} 


\section{Introduction}

The search for physics beyond the Standard Model relies on quantitative theoretical calculations of backgrounds due to known physics. Uncovering signals of new physics requires a good quantitative understanding of the backgrounds as well as the corresponding theoretical uncertainties. The challenge of performing the required theoretical calculations increases with the increasing jet multiplicities used in many search strategies. We are thus encouraged to look for features of the relevant Standard-Model processes that can simplify calculations at higher multiplicities.

In this contribution, we study one of the benchmark Standard-Model processes, production of a $W$ electroweak vector boson accompanied by multiple jets. The short-distance matrix element can be computed systematically in perturbative QCD. A leading-order (LO) calculation leaves a strong dependence on the renormalization and factorization scales introduced in order to define the coupling $\alpha_{s}$ and the parton distribution functions (PDFs). This unphysical dependence becomes stronger with an increasing number of accompanying jets. A next-to-leading order (NLO) calculation is required to obtain a quantitatively reliable prediction. We expect such predictions to be accurate to $10-15 \%$. Future improvements to experimental uncertainties will demand that theorists go to yet higher order in the perturbative expansion.

With the BLACKHAT software library [四], building on the progress in NLO calculations in

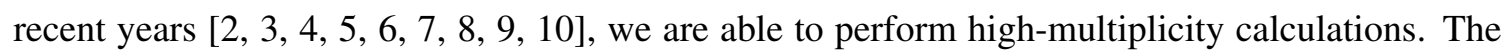
software library uses on-shell methods to supply one-loop amplitudes. In this approach, the amplitude is written as a sum over known integrals,

$$
\text { Amplitude }=\sum_{j \in \text { Basis }} c_{j} \text { Int }_{j}+\text { Rational }
$$

where the integrals are the usual box, triangle, and bubble one-loop integrals. The integrals' coefficients $c_{j}$ as well as the additional rational terms are rational functions of spinor variables. They are built out of Lorentz-invariant bilinear products of spinors. The external momenta are quadratic functions of these spinor variables, and Lorentz invariants are in turn quadratic functions of Lorentz-invariant spinor products. The BLACKHAT library embodies an automated, numerical implementation of on-shell methods, and the unitarity method in particular. The NLO calculation as a whole, including phase-space generation, multichannel integration, and subprocess management, is managed by SHERPA [W]. In addition, the real-emission contributions, with subtractions according to the Catani-Seymour scheme [ए2] as modified by an $\alpha_{\text {dipole }}$ cut-off parameter [ए3], are produced using the COMIX library [प4] (itself a part of SHERPA).

We have used RoOT $n$-tuple files that record phase-space configurations along with their weights, split up into the coefficients of the different functional dependences on $\mu_{\mathrm{R}, \mathrm{F}}$ and the PDFs [45]. This technology is discussed elsewhere in these proceedings.

\section{Jet-Production Ratios}

The transverse momentum distributions in $W+4$ - and $W+5$-jet production are shown in fig. 2 in ref. [ए]] and fig. 6 in ref. [[1]] respectively. The calculations show greatly reduced scale dependence at NLO compared to LO. The NLO corrections soften the distribution for all jets except 


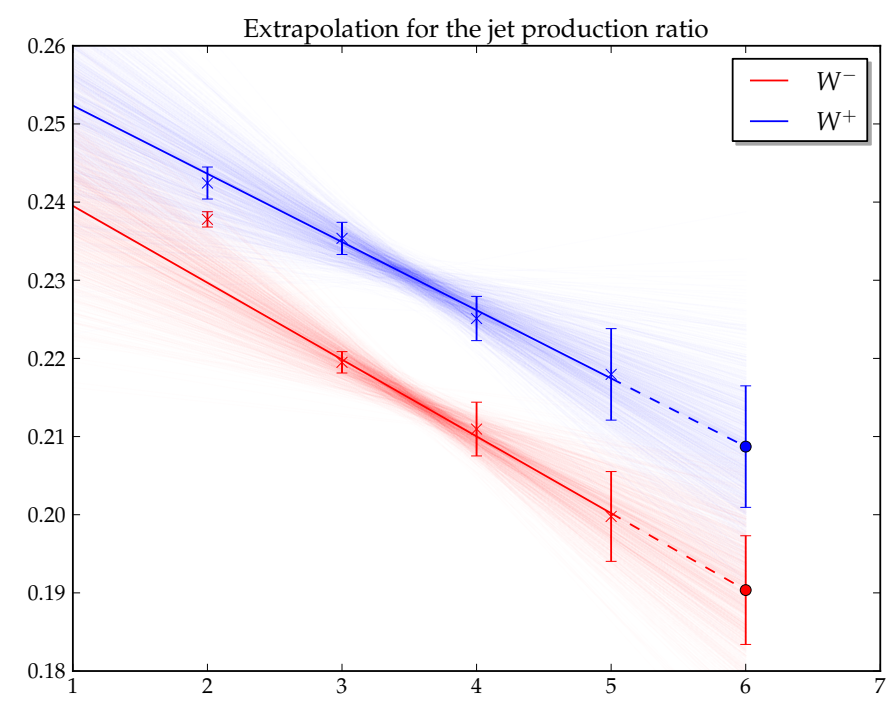

Figure 1: Extrapolations of the ratio of total cross sections at NLO. The lower (red) line shows the extrapolation for the $W^{-}+n / W^{-}+(n-1)$ ratio, and the upper (blue) line for the $W^{+}+n / W^{+}+(n-1)$ ratio. The fainter lines illustrate the uncertainty envelope based on the statistical uncertainty of each underlying cross section.

the softest jet. Successive jet distributions become steeper and steeper. This can be understood as reflecting the greater increase of the partonic center-of-mass invariant $\hat{s}$ when increasing (say) the fifth jet's $p_{\mathrm{T}}$ as opposed to the third jet's (all jets are ordered in decreasing $p_{\mathrm{T}}$ ). The increase in $\hat{s}$ in turn decreases the matrix element as well as the PDFs. Other than the increasingly steep fall, distributions for the third-softest jet and softer jets resemble each other. This raises the question of whether one can think of these jets as 'generic', and whether one can find patterns in $W+n$-jet production.

In order to examine patterns in $W+$ multijet production in greater detail, it is helpful to examine ratios of observable quantities in $W+n$-jet production to that in $W+(n-1)$-jet production. Such ratios should be less sensitive to experimental uncertainties: the luminosity uncertainty should cancel, and the jet-energy scale dependence will be lessened. They should also be less sensitive to theoretical uncertainties: though it is hard to quantify, the scale sensitivity should diminish; and the dependence on the PDFs should decrease as well.

At low multiplicities, however, we expect deviations from generic behavior. In $W+1$-jet production, some subprocesses are missing at LO; and even at NLO, there are strong kinematic restrictions on the phase space of jets. In $W+2$-jet production, there are strong kinematic restrictions on phase space (the $W$ boson cannot be near the leading jet, for example), which only start being relaxed at NLO. In the $W p_{\mathrm{T}}$ distribution, this is reflected in large NLO corrections beyond low $p_{\mathrm{T}}$ in the $W+3$-jet/ $W+2$-jet ratio, while corrections to the $W+n$-jet/ $W+(n-1)$-jet ratio for $n \geq 4$ are more modest.

The simplest pattern we can seek is in the ratio of total cross sections subject to standard jet cuts. (The cuts we use are given in ref. [ए]].) In fig. 四, we show a linear fit to the cross-section 

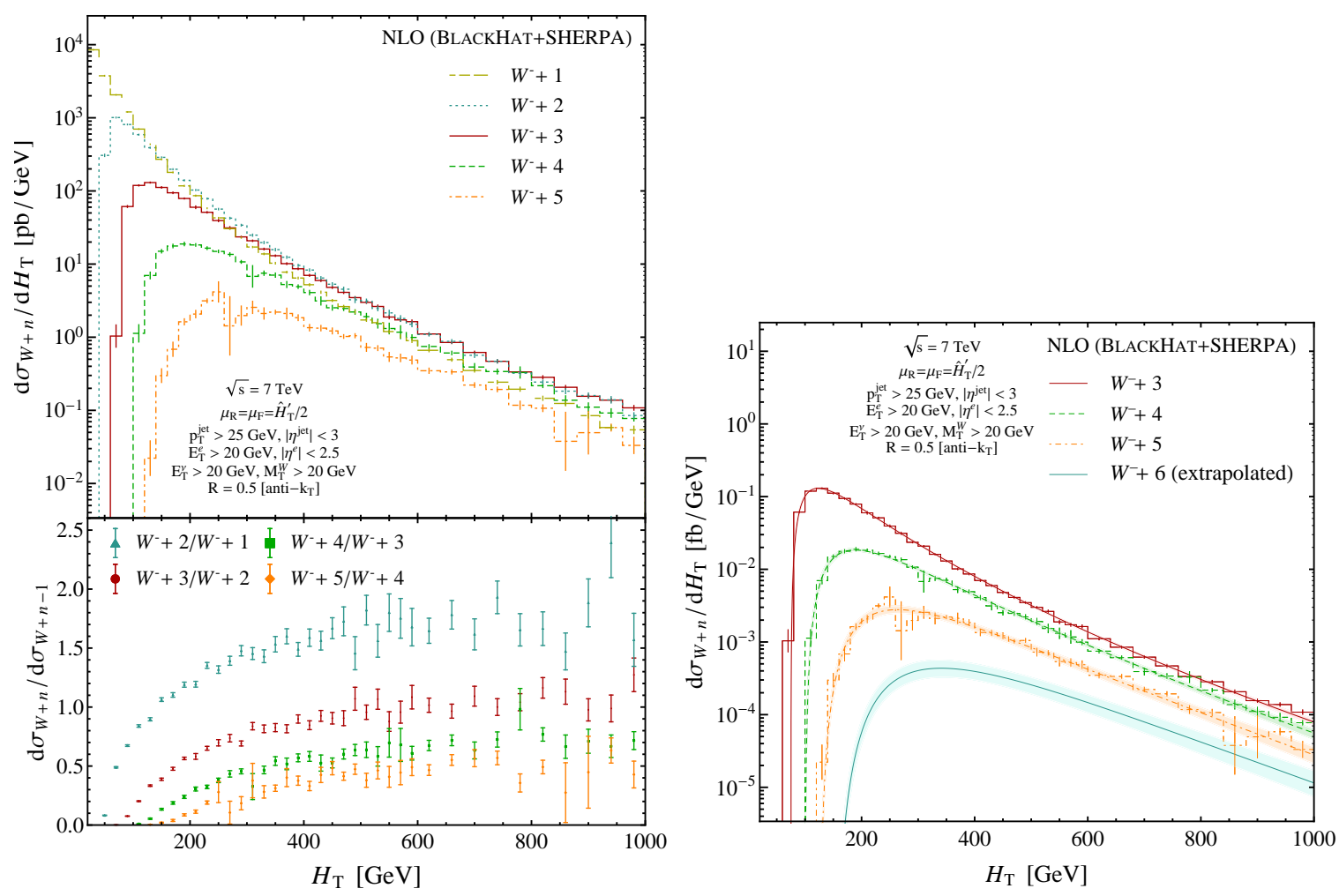

Figure 2: (a) In the upper panel at left, the computed $H_{\mathrm{T}}^{\text {jets }}$ distributions at NLO for $W^{-}$production accompanied by up to five jets; in the lower panel, ratios of these distributions (b) At right, the computed $H_{\mathrm{T}}^{\text {jets }}$ distributions at NLO for $W^{-}$production with three to five accompanying jets, compared with an ratio-based parametrization, along with the expolation to $W^{-}+6$-jet production.

ratios. The fit to the $W+3$-jet/ $W+2$-jet, $W+4$-jet/ $W+3$-jet, and $W+5$-jet/ $W+4$-jet ratios is very good. The $W+5$-jet calculation is needed to make this assessment meaningful; were it absent, we might even be misled into including the $W+2$-jet/ $W+1$-jet ratio. As can be seen in the figure, and as expected, that ratio (at least for $W^{-}$) is quite different from what would be expected from the fit. The linear fit allows us to predict the $W+6$-jet/ $W+5$-jet ratio, and that in turn, allows us give a prediction [17]] for the $W+6$-jet production cross section,

$$
\begin{array}{ll}
W^{-}+6 \text { jets: } & 0.15 \pm 0.01 \mathrm{pb}, \\
W^{+}+6 \text { jets: } & 0.30 \pm 0.03 \mathrm{pb},
\end{array}
$$

where the uncertainty estimates include only statistical uncertainties.

\section{Extrapolating the $H_{\mathrm{T}}^{\text {jets }}$ Distribution}

The total transverse energy in jets, $H_{\mathrm{T}}^{\mathrm{jets}}$, is a good probe into possible new physics at the very highest center-of-mass energies accessible to the LHC. We have computed this distribution for a $W$ boson accompanied by up to five jets. The results are shown in the upper panel of fig. D (a). We may try to predict the distribution for $W+6$-jet production by extrapolating these results. The 
$H_{\mathrm{T}}^{\mathrm{jets}}$ distributions have a threshold, due to the minimum jet transverse momentum. Combined with the decrease towards larger $H_{\mathrm{T}}^{\text {jets }}$, due to the decreasing matrix elements and the falling parton distributions, this threshold qleads to the appearance of a peak in the distribution. The threshold, and hence the peak locations, are different for different numbers of jets. This makes a simple extrapolation at each different value of $H_{\mathrm{T}}^{\text {jets }}$ problematic. Instead, we seek to fit a functional form.

At small $H_{\mathrm{T}}^{\text {jets }}$, we might expect the integral we are evaluating for $n$ jets to have the following form,

$$
\left(\int \frac{d E}{E} g(E)\right)^{m}
$$

where $g(E)$ is slowly varying, and where $m<n$ because not all jets can be soft. This form suggests a functional form of $\ln ^{\tau} \rho$ to include as a factor, where $\rho=H_{\mathrm{T}}^{\text {jets }} /\left(n p_{\mathrm{T}}^{\min }\right)$. We take $\tau$ as a fit parameter. At very large $H_{\mathrm{T}}^{\text {jets }}$ (well to the right of the plots in fig. $\mathbb{Z}$ ), phase space becomes constrained, suggesting the use of a factor like $\left(1-H_{\mathrm{T}}^{\mathrm{jets}} / H_{\mathrm{T}}^{\max }\right)^{\gamma}$, where $H_{\mathrm{T}}^{\max } \simeq 7 \mathrm{TeV}$, and $\gamma$ is a fit parameter. We have previously seen [ए8] that such a factor is appropriate for the Tevatron, where it is more noticeable because of the lower center-of-mass energy.

This leads to the following fit form,

$$
\frac{d \sigma^{W+n}}{d H_{\mathrm{T}}^{\mathrm{jets}}}=a_{s}^{n} N_{n} \ln ^{\tau_{n}} \rho_{n}\left(1-H_{\mathrm{T}}^{\mathrm{jets}} / H_{\mathrm{T}}^{\max }\right)^{\gamma_{n}},
$$

where $N_{n}$ is a normalization, and $a_{s} \equiv \alpha_{s}\left(H_{\mathrm{T}}^{\mathrm{jets}}\right) N_{c} / \pi$. Using this form gives a poor fit to the $H_{\mathrm{T}}^{\mathrm{jets}}$ distributions themselves, but an excellent fit for the ratios of these distributions shown in the bottom panel of fig. $\mathbb{Z}(\mathrm{a})$. We can then fit for the values of the $\tau$ and $\gamma$ parameters; once again, we get a good linear fit. Using a fit with additional parameters to the $W+2$-jet $H_{\mathrm{T}}^{\text {jets }}$ distribution, we can then use predicted values of the $\tau$ and $\gamma$ parameters to obtain predictions for the $H_{\mathrm{T}}^{\text {jets }}$ distribution in $W+n$-jet production. The normalization $N_{n}$ is related to the total cross section; we can solve for it by integrating the fit form using the extrapolated values of $\tau$ and $\gamma$, and comparing with the extrapolated value of the total cross section as in eq. ([R.T). We can cross check this procedure by comparing the 'predicted' curves for $W^{-}+3$ - through $W^{-}+5$-jet production with the directly computed values; this comparison is shown in fig. $\square\left(\right.$ (b). The predicted $H_{\mathrm{T}}^{\text {jets }}$ distribution in $W^{-}+6-$ jet production is shown in the same figure.

\section{Conclusions}

The production of an electroweak vector boson accompanied by multiple jets is an important Standard-Model process at the LHC. We have studied this process in a wide range of kinematic regimes, and with a varying number of accompanying jets. We see indications of simple and regular behavior in NLO calculations of cross sections and distributions beginning with three accompanying jets. We have used this behavior to predict the total cross section and a key distribution in $W+6$-jet production.

\section{Acknowledgments}

This research was supported by the US Department of Energy under contracts DE-AC0276SF00515 and DE-SC0009937. DAK's research is supported by the European Research Council 
under Advanced Investigator Grant ERC-AdG-228301. DM's work was supported by the Research Executive Agency (REA) of the European Union under the Grant Agreement number PITNGA-2010-264564 (LHCPhenoNet). SH's work was partly supported by a grant from the US LHC Theory Initiative through NSF contract PHY-0705682. This research used resources of Academic Technology Services at UCLA.

\section{References}

[1] C. F. Berger, Z. Bern, L. J. Dixon, F. Febres Cordero, D. Forde, H. Ita, D. A. Kosower and D. Maître, Phys. Rev. D 78, 036003 (2008) [arXiv:0803.4180 [hep-ph]]; C. F. Berger, Z. Bern, L. J. Dixon, F. Febres Cordero, D. Forde, T. Gleisberg, H. Ita, D. A. Kosower and D. Maître, Phys. Rev. Lett. 102, 222001 (2009) [arXiv:0902.2760 [hep-ph]].

[2] Z. Bern, L. J. Dixon, D. C. Dunbar and D. A. Kosower, Nucl. Phys. B 425, 217 (1994) [hep-ph/9403226]; Nucl. Phys. B 435, 59 (1995) [hep-ph/9409265].

[3] Z. Bern and A. G. Morgan, Nucl. Phys. B 467, 479 (1996) [hep-ph/9511336]; Z. Bern, L. J. Dixon, D. C. Dunbar and D. A. Kosower, Phys. Lett. B 394 (1997) 105 [hep-th/9611127]; C. Anastasiou, R. Britto, B. Feng, Z. Kunszt and P. Mastrolia, Phys. Lett. B 645 (2007) 213 [hep-ph/0609191]; R. Britto and B. Feng, JHEP 0802, 095 (2008) [arXiv:0711.4284 [hep-ph]]; W. T. Giele, Z. Kunszt and K. Melnikov, JHEP 0804, 049 (2008) [arXiv:0801.2237 [hep-ph]]; R. Britto, B. Feng and P. Mastrolia, Phys. Rev. D 78, 025031 (2008) [arXiv:0803.1989 [hep-ph]]; R. K. Ellis, W. T. Giele, Z. Kunszt and K. Melnikov, Nucl. Phys. B 822 (2009) 270 [arXiv:0806.3467 [hep-ph]].

[4] Z. Bern, L. J. Dixon and D. A. Kosower, Nucl. Phys. B 513, 3 (1998) [hep-ph/9708239].

[5] R. Britto, F. Cachazo and B. Feng, Nucl. Phys. B 725, 275 (2005) [hep-th/0412103].

[6] R. Britto, F. Cachazo, B. Feng and E. Witten, Phys. Rev. Lett. 94, 181602 (2005) [hep-th/0501052].

[7] C. F. Berger, Z. Bern, L. J. Dixon, D. Forde and D. A. Kosower, Phys. Rev. D 74, 036009 (2006) [hep-ph/0604195].

[8] G. Ossola, C. G. Papadopoulos and R. Pittau, Nucl. Phys. B 763, 147 (2007) [hep-ph/0609007].

[9] D. Forde, Phys. Rev. D 75, 125019 (2007) [arXiv:0704.1835 [hep-ph]].

[10] S. D. Badger, JHEP 0901, 049 (2009) [arXiv:0806.4600 [hep-ph]].

[11] T. Gleisberg, S. Höche, F. Krauss, A. Schalicke, S. Schumann and J. C. Winter, JHEP 0402, 056 (2004) [hep-ph/0311263]; T. Gleisberg, S. Höche, F. Krauss, M. Schönherr, S. Schumann, F. Siegert and J. Winter, JHEP 0902, 007 (2009) [arXiv:0811.4622 [hep-ph]].

[12] S. Catani and M. H. Seymour, Nucl. Phys. B 485, 291 (1997) [Erratum-ibid. B 510, 503 (1998)] [hep-ph/9605323].

[13] Z. Nagy, Phys. Rev. D 68, 094002 (2003) [hep-ph/0307268].

[14] T. Gleisberg and S. Höche, JHEP 0812, 039 (2008) [arXiv:0808.3674 [hep-ph]].

[15] Z. Bern, L. J. Dixon, F. Febres Cordero, S. Höche, H. Ita, D. A. Kosower and D. Maitre, Comput. Phys. Commun. 185, 1443 (2014) [arXiv:1310.7439 [hep-ph]].

[16] C. F. Berger, Z. Bern, L. J. Dixon, F. Febres Cordero, D. Forde, T. Gleisberg, H. Ita, D. A. Kosower and D. Maître, Phys. Rev. Lett. 106, 092001 (2011) [arXiv:1009.2338 [hep-ph]]. 
[17] Z. Bern, L. J. Dixon, F. Febres Cordero, S. Hoeche, H. Ita, D. A. Kosower, D. Maitre and K. J. Ozeren, Phys. Rev. D 88, 014025 (2013) [arXiv:1304.1253 [hep-ph]].

[18] C. F. Berger, Z. Bern, L. J. Dixon, F. Febres Cordero, D. Forde, T. Gleisberg, H. Ita, D. A. Kosower and D. Maitre, Phys. Rev. D 82, 074002 (2010) [arXiv:1004.1659 [hep-ph]]. 